\title{
A Case of Pancreatic Schwannoma Diagnosed Preoperatively by Endoscopic Ultrasonography-Guided Fine Needle Aspiration and Treated with Laparoscopic Surgery
}

\author{
Kazuao Hayashi, ${ }^{1}$ Atsunori Tsuchiya, ${ }^{1, *}$ Satoshi Ikarashi, ${ }^{1}$ Kazuyasu Takizawa, ${ }^{2}$ and Shuji Terai
}

\begin{abstract}
Background: Pancreatic tumors are often difficult to diagnose in atypical cases, and a pancreatic schwannoma is very rare. We present a case of pancreatic schwannoma with calcification diagnosed preoperatively by endoscopic ultrasonography (EUS)-guided fine needle aspiration (FNA) and treated with laparoscopic distal pancreatectomy. Presentation: A 72-year-old-woman was admitted to our hospital due to a $6 \times 4.5 \mathrm{~cm}$ large tumor in the pancreatic tail. Imaging modalities revealed that the tumor was hypovascular and gradually enhanced with calcification, but was without cystic lesions. EUS revealed the tumor had a clear boundary with a low echoic mass. EUSFNA was performed and spindle-shaped cells that were immunopositive for S-100 and negative for c-kit, CD34, and desmin were detected, resulting in a diagnosis of schwannoma. Laparoscopic distal pancreatectomy with splenectomy was safely performed without recurrence for a year.

Conclusions: Schwannoma is very rare; however, characteristics of the tumor, such as calcification, can help the diagnosis and, if possible, EUS-FNA should be performed for an appropriate treatment decision.
\end{abstract}

Keywords: schwannoma; calcification; endoscopic ultrasonography-guided fine needle aspiration; S-100, laparoscopic surgery

\section{Introduction and Background}

Pancreatic tumor is often difficult to diagnose in atypical cases. Pancreatic schwannoma is a very rare tumor; $<50$ cases of pancreatic schwannoma have been described in the English literature for the past 30 years. ${ }^{1}$ Here we present a case of pancreatic schwannoma with calcification and without cystic lesions that was diagnosed preoperatively by endoscopic ultrasonography (EUS)-guided fine needle aspiration (FNA) and treated with laparoscopic surgery.

\section{Presentation of Case}

A 72-year-old-woman was admitted to our hospital with a $6 \times 4.5 \mathrm{~cm}$ huge tumor in which an artery was penetrating in the pancreatic tail (Fig. 1A, white arrows). She had a history of hypertension, spinal canal stenosis, and inappropriate antidiuretic hormone syndrome. She had no abdominal symptoms; however, survey for malignancy helped detect the tumor incidentally. Laboratory data revealed a low white blood cell count of 2440/ $\mu \mathrm{L}$ (normal range [NR]: 3300-8600) and low sodium level (133 mEq/dL, NR: 138-145). The following tumor markers were all within the respective NRs: carcinoembryonic antigen $(4.1 \mathrm{ng} / \mathrm{mL}, \mathrm{NR}$ : $\leqq 5.8)$; carbohydrate antigen $19-9(12 \mathrm{U} / \mathrm{mL}, \mathrm{NR}: \leqq 37)$; duke pancreatic antigen type $2(<25 \mathrm{U} / \mathrm{mL}, \mathrm{NR}: \leqq 150)$; and s-pancreas antigen-1 $(8.5 \mathrm{U} / \mathrm{mL}, \mathrm{NR}$ : $\leqq 30)$. Contrastenhanced computed tomography revealed calcification

Divisions of ${ }^{1}$ Gastroenterology and Hepatology and ${ }^{2}$ Digestive and General Surgery, Graduate School of Medical and Dental Sciences, Niigata University, Niigata, Japan.

*Address correspondence to: Atsunori Tsuchiya, PhD, MD, Division of Gastroenterology and Hepatology, Graduate School of Medical and Dental Sciences, Niigata University, 1-757 Asahimachi-dori, Chuo-ku, Niigata 951-8510, Japan, E-mail: atsunori@med.niigata-u.ac.jp

(C) Kazuao Hayashi et al. 2018; Published by Mary Ann Liebert, Inc. This Open Access article is distributed under the terms of the Creative Commons License (http://creativecommons.org/licenses/by/4.0), which permits unrestricted use, distribution, and reproduction in any medium, provided the original work is properly cited. 

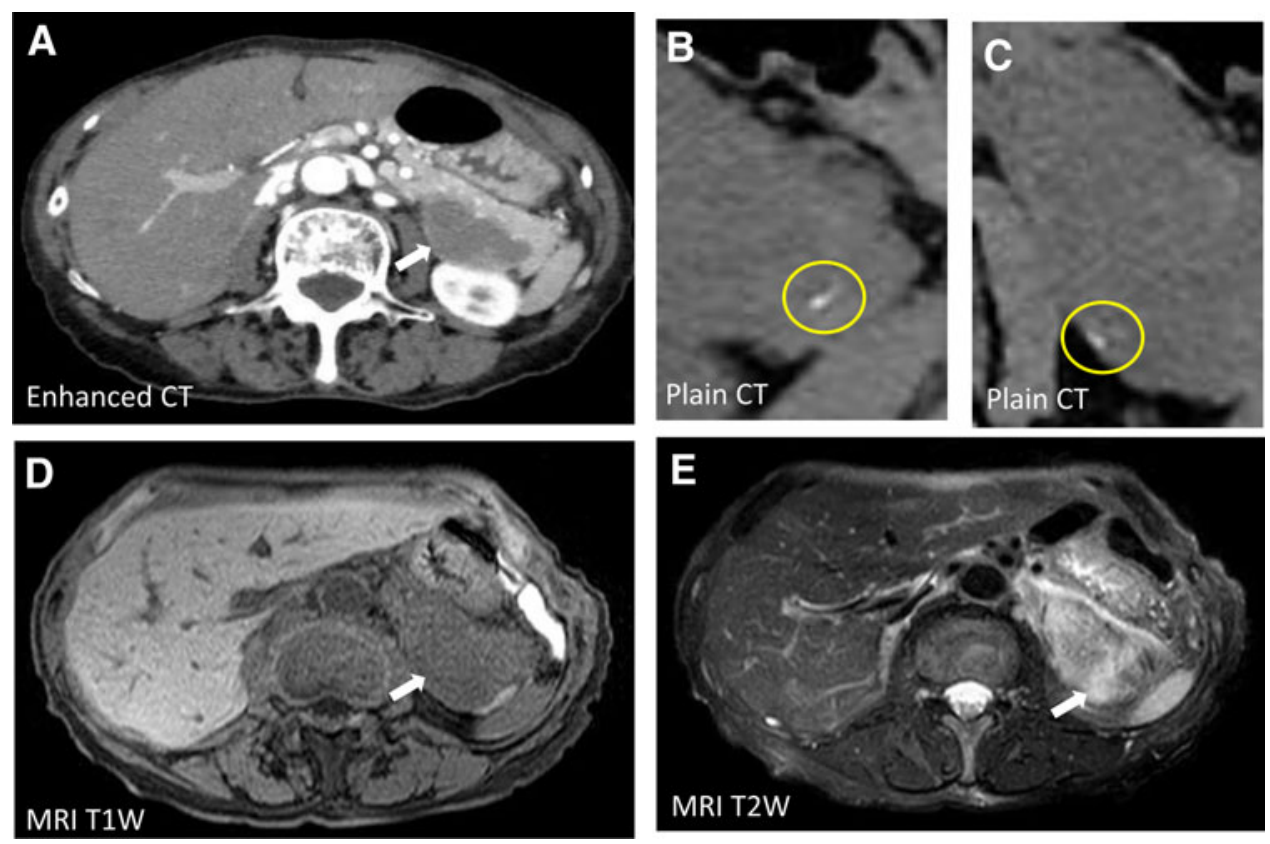

FIG. 1. CT and MRI. (A) CT revealed a 6-cm hypovascular tumor in the pancreatic tail, indicated by white arrow. (B, C) Calcification was detected at the edge of the tumor. Yellow circles indicate calcification.

(D, E) MRI showed hypointensity of the tumor on T1-weighted images (D), and hyperintensity on T2-weighted images (E); tumor indicated by white arrows. CT, computed tomography; MRI, magnetic resonance imaging.

at the edge of the tumor (Fig. 1B, C), no cystic lesion, and gradual enhancement. Magnetic resonance imaging revealed hypointensity of the tumor on T1-weighted images (Fig. 1D) and hyperintensity on T2-weighted images (Fig. 1E). EUS revealed the tumor had a clear boundary with a low echoic mass. We suspected that it was a neuroendocrine tumor, acinar cell carcinoma, solitary-pseudopapillary neoplasm, schwannoma, or anaplastic ductal carcinoma based on the calcification, gradual enhancement, and expansive growth. Therefore, EUS-FNA was performed (Fig. 2A), and spindleshaped cells (Fig. 2B, C) that were immunopositive for S-100 (Fig. 2D) and negative for c-kit, CD34, and desmin were detected. Therefore, the final diagnosis was schwannoma. Laparoscopic distal pancreatectomy with splenectomy was performed and the patient is currently well without recurrence for 1 year.

\section{Discussion}

Schwannomas are neurogenic neoplasms derived from Schwann cells of the peripheral nerve sheaths. Schwannoma is often detected in the head and neck, extremities, mediastinum, and retroperitoneum; however, it is very rare in the pancreas. ${ }^{2}$ Schwannoma often contains a solid component with areas of degenerative changes such as a cyst, calcification, hemorrhage, and hyalinization. $^{3}$ In this case, calcification at the edge of the tumor was detected; therefore, when calcification is detected in the tumor, a diagnosis of schwannoma should be considered. Precise preoperative diagnosis of a pancreatic schwannoma is challenging because the clinical symptoms and radiological characteristics of schwannomas are nonspecific. Schwannomas have characteristic spindle cells with myxoid stroma, and are immunopositive for S-100 (differential diagnosis; Table 1). ${ }^{1,2,5-8}$ The majority of schwannomas are benign; however, malignant cases that often have cystic formation and/or large tumor size (5 of 47 cases; $10.6 \%$ ) are sometimes reported. ${ }^{2}$ An appropriate diagnosis regarding the potential for malignancy has to be performed before a treatment decision can be made. In our case, the tumor was relatively huge and hypovascular, thus we cannot exclude the malignant potential of the tumor; however, EUS-FNA definitively diagnosed the schwannoma and estimated the malignant potential, after which a minimally invasive laparoscopic distal pancreatectomy with splenectomy was performed without 

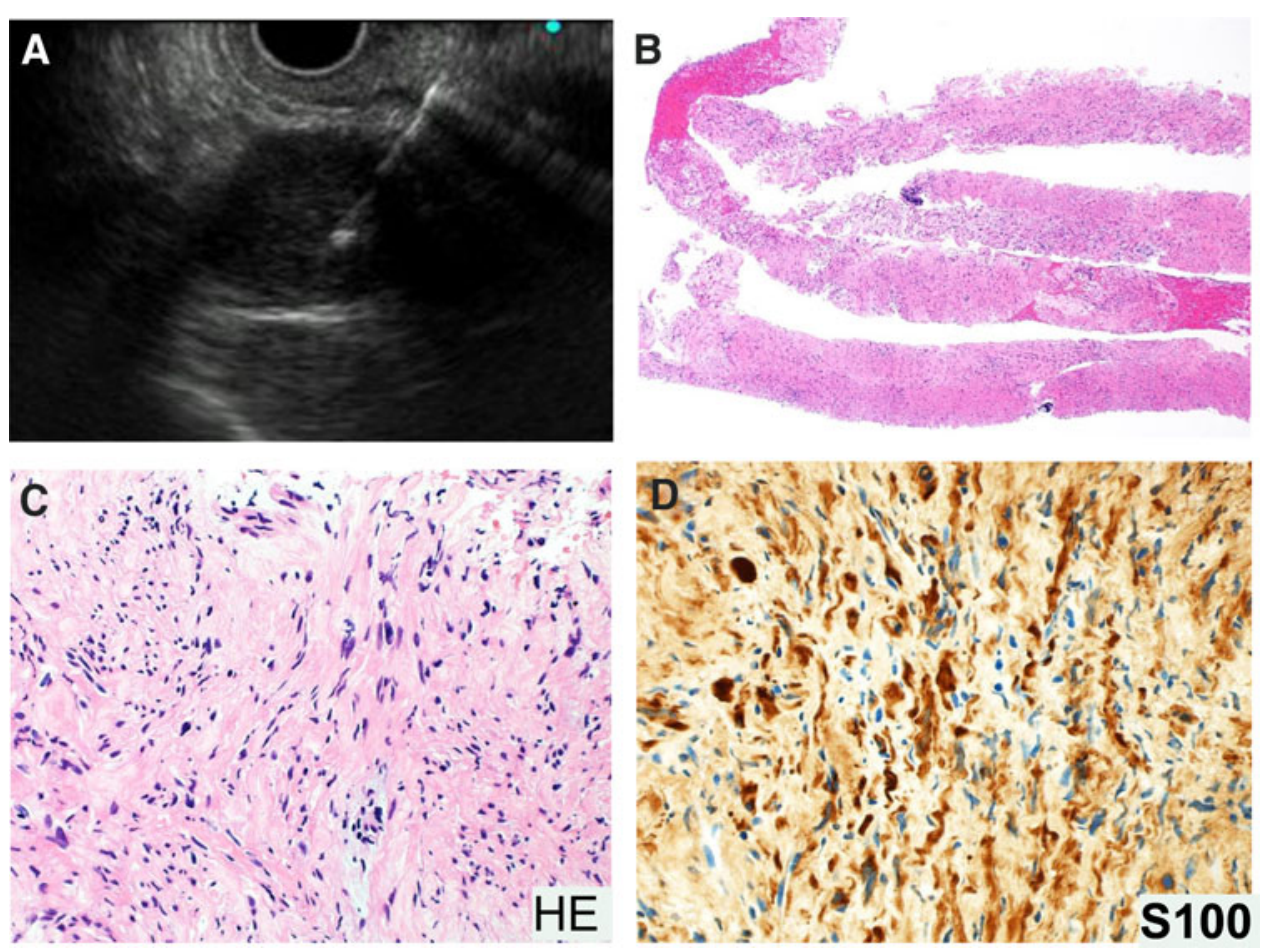

FIG. 2. EUS-FNA and histological analysis. (A) EUS-FNA was performed against the clear boundary of the low echoic mass. (B-D) Spindle-shaped cells were detected by hematoxylin-eosin staining (B: original magnification $\times 40$, and C: original magnification $\times 200$ ); these cells were positive for S-100 (D: original magnification $\times 200$ ). EUS-FNA, endoscopic ultrasonography-guided fine needle aspiration; HE, hematoxylin and eosin stain.

recurrence. If the biopsy revealed that the primary tumor was a malignant schwannoma or any other malignant tumor, we selected laparotomy as the surgical procedure. EUS-FNA can often obtain only very small specimens, and there have been some reports stating that only a small proportion of histologically proven schwannomas can be diagnosed correctly using EUS-FNA, which imposes a massive limitation. ${ }^{4}$ However, in this case, EUS-
FNA did provide an accurate diagnosis, resulting in an appropriate treatment. Development of a new EUS-FNA device would be possible to achieve more accurate diagnosis.

\section{Conclusion}

Schwannoma is a very rare tumor; however, characteristics identified in imaging studies, such as calcification, hemorrhage, and hyalinization, can help make

Table 1. Differential Diagnosis of Schwannoma

\begin{tabular}{|c|c|c|c|c|}
\hline & Schwannoma & NET & $\mathrm{ACC}$ & SPN \\
\hline \multicolumn{5}{|l|}{ Imaging technique } \\
\hline Enhanced $\mathrm{CT}$ & $\begin{array}{l}\text { Well-defined hypovascular } \\
\text { tumor with delayed } \\
\text { enhancement }\end{array}$ & Well-defined hypovascular tumor & $\begin{array}{l}\text { Well-defined iso-hypovascular } \\
\text { tumor }\end{array}$ & $\begin{array}{l}\text { Well-defined } \\
\text { hypovascular tumor }\end{array}$ \\
\hline MRI & T1 low/T2 high & T1 low/T2 high & T1 low/T2 iso-high & T1/T2: low-high-mix \\
\hline Pathology & $\begin{array}{l}\text { Spindle-shaped cells with } \\
\text { myxoid stroma }\end{array}$ & Trabecular/pseudorosette structure & Acinar structure & Pseudopapillary structure \\
\hline \multicolumn{5}{|l|}{$\mathrm{IHC}$} \\
\hline Positive makers & S-100, vimentin & $\begin{array}{l}\text { CD56, chromogranin A, } \\
\text { synaptophysin }\end{array}$ & Trypsin, BCL10 & $\beta$-catenin, vimentin \\
\hline
\end{tabular}

NET, neuroendocrine tumor; ACC, acinar cell carcinoma; SPN, solitary-pseudopapillary neoplasm; CT, computed tomography; IHC, immunohistochemistry; MRI, magnetic resonance imaging. 
an accurate diagnosis. Furthermore, the potential for malignancy needs to be estimated to provide appropriate treatment. Therefore, if possible, EUS-FNA should be performed before a treatment decision is made.

\section{Acknowledgments}

The authors thank Masahiro Ogawa, Junji Kohisa, Kazuya Takahashi, Kentarou Tominaga, Ken-ichi Mizuno, Satoru Hashimoto, Junji Yokoyama, and Satoshi Yamagiwa (Division of Gastroenterology and Hepatology, Niigata University), Annenkov Alexey (Center for Transdisciplinary Research, Niigata University), Kaori Takamura and Yoichi Ajioka (Division of Molecular and Diagnostic Pathology, Niigata University), and Toshifumi Wakai (Division of Digestive and General Surgery, Niigata University) for their cooperation.

\section{Author Disclosure Statement}

No competing financial interests exist.

\section{Author Contributions}

All authors are doctors in charge and all authors contributed in the writing of this article.

\section{References}

1. Moriya T, Kimura W, Hirai I, et al. Pancreatic schwannoma: case report and an updated 30-year review of the literature yielding 47 cases. World $J$ Gastroenterol. 2012;18:1538-1544.
2. Suzuki S, Kaji S, Koike N, et al. Pancreatic schwannoma: a case report and literature review with special reference to imaging features. JOP. 2010;11: 31-35.

3. Ohbatake $\mathrm{Y}$, Makino I, Kitagawa $\mathrm{H}$, et al. A case of pancreatic schwannoma-the features in imaging studies compared with its pathological findings: report of a case. Clin J Gastroenterol. 2014;7: 265-270.

4. Abu-Zaid A, Azzam A, Abou Al-Shaar H, et al. Pancreatic tail schwannoma in a 44-year-old male: a case report and literature review. Case Rep Oncol Med. 2013;2013:416713.

5. Ohara Y, et al. Pancreatic neuroendocrine tumor and solid-pseudopapillary neoplasm: Key immunohistochemical profiles for differential diagnosis. World J Gastroenterol. 2016;22:8596-8604.

6. Hsu MY, et al. CT and MRI features of acinar cell carcinoma of the pancreas with pathological correlations. Clin Radiol. 2010;65:223-229.

7. La Rosa S, et al. Clinicopathologic study of 62 acinar cell carcinomas of the pancreas: insights into the morphology and immunophenotype and search for prognostic markers. Am J Surg Pathol. 2012;36:1782-1795.

8. Vassos N, et al. Solid-pseudopapillary neoplasm (SPN) of the pancreas: case series and literature review on an enigmatic entity. Int J Clin Exp Pathol. 2013;6:1051-1059.

Cite this article as: Hayashi K, Tsuchiya A, Ikarashi S, Takizawa K, Terai $S$ (2018) A case of pancreatic schwannoma diagnosed preoperatively by endoscopic ultrasonography-guided fine needle aspiration and treated with laparoscopic surgery, Journal of Pancreatic Cancer 4:1, 7-10, DOI: 10.1089/pancan.2018.0002.

$\begin{aligned} & \text { Abbreviations Used } \\ & \mathrm{CT}=\text { computed tomography } \\ & \mathrm{EUS}=\text { endoscopic ultrasonography } \\ & \mathrm{FNA}=\text { fine needle aspiration } \\ & \mathrm{MRI}=\text { magnetic resonance imaging }\end{aligned}$

